TITLE:

Irregular parameter dependence of generalized diffusion coefficients based on large deviation statistical analysis

$\operatorname{AUTHOR}(\mathrm{S})$ :

Yoshida, M; Miyazaki, S; Fujisaka, H

CITATION:

Yoshida, M ... [et al]. Irregular parameter dependence of generalized diffusion coefficients based on large deviation statistical analysis. Physical Review E 2006, 74(2): 026204.

ISSUE DATE:

2006-08

URL:

http://hdl.handle.net/2433/50286

RIGHT:

Copyright 2006 American Physical Society 
PHYSICAL REVIEW E 74, 026204 (2006)

\title{
Irregular parameter dependence of generalized diffusion coefficients based on large deviation statistical analysis
}

\author{
Masaomi Yoshida, ${ }^{*}$ Syuji Miyazaki, ${ }^{\dagger}$ and Hirokazu Fujisaka ${ }^{\ddagger}$ \\ Department of Applied Analysis and Complex Dynamical Systems, Graduate School of Informatics, \\ Kyoto University, Kyoto 606-8501, Japan \\ (Received 24 June 2005; revised manuscript received 22 June 2006; published 3 August 2006)
}

\begin{abstract}
The nonperturbative non-Gaussian characteristics of diffusive motion are examined in the framework of the large deviation statistical theory, where simple extended mapping models showing chaotic diffusion are taken as an example. Furthermore, by rigorously solving the large deviation statistical quantities, it is found that the same type of anomalous, complex control parameter dependence as that for the diffusion coefficient reported by Klages and Dorfman is also observed in the large deviation statistical quantities such as the weighted average, the generalized diffusion coefficient, and the generalized power spectrum densities.
\end{abstract}

DOI: 10.1103/PhysRevE.74.026204 PACS number(s): 05.45. $-\mathrm{a}, 05.60 .-\mathrm{k}, 02.50 .-\mathrm{r}, 05.40 .-\mathrm{a}$

\section{INTRODUCTION}

Diffusion is one of the most important and widely observed phenomena, not only in statistical and chemical physics but also in other fields of science and engineering. For a statistically steady random variable $v_{t}$, the variable $y_{t}$ generated by the dynamics

$$
y_{t+1}=y_{t}+v_{t} \quad(t=0,1,2,3, \ldots)
$$

shows a diffusive motion. Namely, the variance of $y_{t}, \sigma_{0}^{2}(t)$ $\equiv\left\langle\left(y_{t}-y_{0}-\langle v\rangle t\right)^{2}\right\rangle,\langle\cdots\rangle$ being the ensemble average of $\cdots$, obeys the law $\sigma_{0}^{2}(t) \approx 2 D t$ for large $t$. The diffusion coefficient $D$ is a key quantity to characterize the statistics of the diffusive process.

The diffusion process is observed also in chaotic dynamics [1]. Namely, if the quantity $v_{t}$ is generated by the chaotic dynamics and has the mixing property, i.e., the time correlation function of $v_{t}$ decays in an appropriately fast way, the quantity $y_{t}$ shows diffusive statistics. This phenomenon is called either deterministic diffusion or chaotic diffusion and is one of the eminent characteristics in nonlinear dynamics.

In order to study diffusive statistics, the diffusion coefficient is quite important. This is because the diffusion coefficient is directly related to the asymptotic form of the maximum region of the probability density for the variable $y_{t}$ as

$$
\begin{gathered}
P_{t}(y) \sim \exp \left[-S\left(\frac{y-y_{0}}{t}\right) t\right], \\
S(v)=\frac{1}{4 D}(v-\langle v\rangle)^{2}
\end{gathered}
$$

for large $t$ [2]. Because of the central limit theorem, this Gaussian form holds even if the variable $v_{t}$ is very different from the Gaussian random variable. The diffusion coefficient $D$ thus cannot describe the non-Gaussian statistics of $y_{t}$ in an

\footnotetext{
*Present address: NS Solutions Corporation, 20-15, Shinkawa 2-chome, Chuo-ku, 104-0033 Tokyo, Japan.

${ }^{\dagger}$ Corresponding author. Electronic address: syuji@i.kyoto-u.ac.jp

‡Electronic address: fujisaka@i.kyoto-u.ac.jp
}

appropriate manner. The non-Gaussian characteristics, which are observed typically in the tail regions of $P_{t}(y)$, are analyzed in the framework of the large deviation statistical theory [2]. This analysis leads to the concept of "fluctuation of diffusion coefficient" [2]. The generalized diffusion coefficient, which is explained in detail in Sec. II, can describe various statistics of $y_{t}$ and $v_{t}$ including nonballistic and ballistic motions in a clear-cut way.

Meanwhile, Klages and Dorfman recently reported a complex, anomalous control parameter dependence of diffusion coefficients, taking simple chaotic mapping dynamics showing diffusive motion (fractal diffusion coefficient) [3]. This complex behavior reflects the existence of infinitely many unstable limit cycles in the state space.

As explained above, there are two interesting problems in the statistical characterization of chaotic diffusion: large deviation characterization and the complex control parameter dependence of statistical quantities for diffusive motion. The fundamental purpose of the present paper is to examine chaotic diffusive motion from the viewpoint of large deviation statistics and the complex control dependence of statistical quantities. In particular, we pay attention to how complex control parameter dependence is observed in large deviation statistical quantities.

The present paper is organized as follows. In Sec. II, we briefly review the formalism of the large deviation statistical analysis of diffusive motion to describe the nonperturbative non-Gaussian characteristics of diffusive motion. Furthermore, the formulas to determine the large deviation statistical quantities in chaotic dynamics are summarized. In Sec. III, taking a simple mapping model to generate chaotic diffusion, we derive analytically the large deviation statistical quantities. In Sec. IV, we study how the control parameter affects the diffusion characteristics such as the conventional diffusion coefficient and the large deviation statistical quantities. It will be found that for the present models a complex control parameter dependence of diffusion coefficients is observed, and we will find that such an anomalous control parameter dependence is observed even with the large deviation statistical quantities. We give summaries and concluding remarks in Sec. V. 


\section{DESCRIPTION OF NON-GAUSSIAN FLUCTUATION}

\section{A. Framework of large-deviation statistics}

Let us briefly describe large deviation statistics following the series of studies by Fujisaka and his co-workers [4-9]. Consider a stationary time series $u$. The average over time interval $T$ is given by this formula,

$$
\bar{u}_{t}=\frac{1}{T} \int_{t}^{t+T} u_{s} d s,
$$

which distributes when $T$ is finite. When $T$ is much larger than the correlation time of $u$, the distribution of coarsegrained $u$ is assumed to be an exponential form $P_{T}(u)$ $\propto e^{-S(u) T}$. Here we can introduce the fluctuation spectrum $S(u)$ as

$$
S(u)=-\lim _{T \rightarrow \infty} \frac{1}{T} \log P_{T}(u) \quad[S(u) \geqslant 0] .
$$

When $T$ is comparable to the correlation time, correlation cannot be ignored, so nonexponential or nonextensive statistics will be a problem, but here we do not discuss this point further. Let $q$ be a real parameter. We introduce the generating function $Z_{q}$ of $T$ by this definition.

$$
Z_{q}(T) \equiv\left\langle e^{q T \bar{u}_{T}}\right\rangle=\int_{-\infty}^{\infty} P_{T}(u) e^{q T u} d u .
$$

We can also here assume the exponential distribution and introduce a characteristic function $\phi(q)$ as

$$
\phi(q)=\lim _{T \rightarrow \infty} \frac{1}{T} \log Z_{q}(T) .
$$

The Legendre transform holds between fluctuation spectrum $S(u)$ and characteristic function $\phi(q)$, which is obtained from saddle-point calculations.

$$
\frac{d S(u)}{d u}=q, \quad \phi(q)=-S[u(q)]+q u(q) .
$$

In this transform a derivative $d \phi / d q$ appears, and it is a weighted average of $\bar{u}_{T}$,

$$
u(q)=\phi^{\prime}(q)=\lim _{T \rightarrow \infty} \frac{\left\langle\bar{u}_{T} e^{q T \bar{u}_{T}}\right\rangle}{Z_{q}(T)} \equiv \lim _{T \rightarrow \infty}\left\langle\bar{u}_{T} ; q\right\rangle_{T}
$$

so we find that $q$ is a kind of weight index. We can also introduce susceptibility $\chi(q)=\frac{d u(q)}{d q}$ as a weighted variance. These statistical structure functions $S(u), \phi(q), u(q), \chi(q)$ constitute the framework of statistical thermodynamics of temporal fluctuation, which characterize static properties of chaotic dynamics. In order to consider dynamic properties, we can introduce this generalized spectrum density as a weighted average of normal spectrum density

$$
I_{q}(\omega)=\lim _{T \rightarrow \infty} \frac{1}{T} \frac{\left\langle\left|\int_{0}^{T}\left[u_{t+s}-u(q)\right] e^{-i \omega s} d s\right|^{2} e^{q T \bar{u}_{T}}\right\rangle}{Z_{q}(T)} .
$$

We note that our characteristic function $\phi(q)$ corresponds to topological pressure in the other literature, and the largedeviation formalism has been discussed by many authors [10-12]. However, we also note that our generalization of time correlation functions and power spectra based on the large-deviation formalism has not been analyzed in the above-mentioned literature.

\section{B. Generalization of diffusion coefficients}

Fujisaka and Inoue applied this large-deviation statistical analysis to diffusion processes [2]. In the following, we restrict $u(q)$ to weighted averages of velocity obtained from a diffusion process. Equation (9) leads to

$$
u^{\prime}(q)=\lim _{t \rightarrow \infty} \frac{1}{t}\left\langle\left[x_{t}-x_{0}-u(q) t\right]^{2} ; q\right\rangle_{t},
$$

where $x_{t}$ denotes position at time $t$. In Eqs. (9) and (10), $u(0)\left[=\phi^{\prime}(0)\right]$ and $u^{\prime}(0)\left[=\phi^{\prime \prime}(0)\right]$ are, respectively, identical to the drift velocity $v_{d}$ and $2 D$, where $D$ is the diffusion coefficient. Therefore $u(q)$ may be regarded as the generalized drift velocity (order- $q$ drift velocity). Furthermore, the generalized variance (order- $q$ variance) defined by

$$
\sigma_{q}^{2}(t) \equiv\left\langle\left[x_{t}-x_{0}-u(q) t\right]^{2} ; q\right\rangle_{t}=t^{2}\left\langle\left[\bar{u}_{t}-u(q) t\right]^{2} ; q\right\rangle_{t}
$$

asymptotically takes the form

$$
\sigma_{q}^{2}(t)=2 D_{q} t
$$

for large $t$, where

$$
D_{q} \equiv \lim _{t \rightarrow \infty} \frac{\sigma_{q}^{2}(t)}{2 t}=\frac{u^{\prime}(q)}{2}=\frac{\chi(q)}{2}=\frac{1}{2 S^{\prime \prime}[u(q)]} .
$$

By introducing the generalized (order- $q$ ) time correlation function by

$$
C_{q}(s)=\lim _{t \rightarrow \infty} \frac{\left\langle\left[u_{s}-u(q)\right]\left[u_{0}-u(q)\right] e^{q t \bar{u}_{t}}\right\rangle}{Z_{q}(t)},
$$

the generalized (order- $q$ ) diffusion coefficient is expressed as

$$
D_{q}=\int_{0}^{\infty} C_{q}(s) d s
$$

where $D_{q=0}$ is identical to the ordinary diffusion coefficient.

Applying the cumulant expansion to $Z_{q}(t), \phi(q)$ is expanded as

$$
\phi(q)=\phi^{\prime}(0) q+\frac{1}{2} \phi^{\prime \prime}(0) q^{2}
$$

provided that $|q|$ is much less than the convergence radius $q_{0}$ of the expansion. This immediately leads to

$$
\begin{gathered}
u(q)=\phi^{\prime}(0)+\phi^{\prime \prime}(0) q, \\
S(u)=\frac{\left[u-\phi^{\prime}(0)\right]^{2}}{2 \phi^{\prime \prime}(0)}(\geqslant 0) .
\end{gathered}
$$

The Gaussian approximation holds for $u$ satisfying $\left|u-\phi^{\prime}(0)\right| / \phi^{\prime}(0) \ll q_{0}$. If $u_{t}$ is Gaussian, the $q_{0}=+\infty$ and Eq. 
(18) holds for any $u$. Therefore the non-Gaussian property causes the $q$-dependence of $D_{q}$, which is the origin of the existence of an infinite number of statistical characteristics of diffusive motion.

\section{Relationship between generalized Frobenius-Perron operators and statistical structure functions for one-dimensional mapping systems}

Let us consider the case of a one-dimensional map. Let $u\left[x_{n}\right]$ be a unique function of $x$, which is governed by the map $x_{n+1}=f\left(x_{n}\right)$. The question is how to obtain statistical structure functions and generalized spectral densities of $u$. The answer is to solve eigenvalue problems of a generalized Frobenius-Perron operator. As we mentioned before, the characteristic function $\phi(q)$ is given by the asymptotic form of the generating function $Z_{q}(n)$ in the limit of $n \rightarrow \infty$ corresponding to the temporal coarse-grained quantity $\bar{u}_{n}$ $=\frac{1}{n} \sum_{j=0}^{n-1} u\left[x_{j+m}\right]$, where we assume an exponential fast decay of time correlations of $u$. A generating function can be expressed in terms of invariant density,

$$
\begin{aligned}
Z_{q}(n) & \equiv\left\langle e^{q n \bar{u}_{n}}\right\rangle=\int \rho^{*}(x) \exp \left[q \sum_{j=0}^{n-1} u\left[f^{j}(x)\right]\right] d x \\
& =\int \mathcal{H}_{q}^{n} \rho^{*}(x) d x,
\end{aligned}
$$

where the generalized Frobenius-Perron operator $\mathcal{H}_{q}$ is defined and related to the original one as

$$
\mathcal{H}_{q} G(x)=\mathcal{H}\left[e^{q u[x]} G(x)\right]=\sum_{j} \frac{e^{q u\left[y_{j}\right]} G\left(y_{j}\right)}{\left|f^{\prime}\left(y_{j}\right)\right|}
$$

for an arbitrary function $G(x)\left(\mathcal{H}_{0}=\mathcal{H}\right)$, where the sum is taken over all solutions $y_{j}(x)$ satisfying $f\left(y_{j}\right)=x$. To obtain the above equation, the following relation is repeatedly used:

$$
\mathcal{H}\left\{G(x)\left[q \sum_{j=0}^{m} u\left[f^{j}(x)\right]\right]\right\}=\left[\mathcal{H}_{q} G(x)\right]\left[q \sum_{j=0}^{m-1} u\left[f^{j}(x)\right]\right] .
$$

Let $\nu_{q}^{(0)}$ be the maximum eigenvalue of $H_{q}$. The characteristic function is given by its logarithm as

$$
\phi(q)=\log \nu_{q}^{(0)} \quad\left(\nu_{0}^{(0)}=1\right) .
$$

The weighted average $u(q)$ and the weighted variance $\chi(q)$ are given by the first and the second derivatives of $\phi(q)$.

The generalized power spectrum is

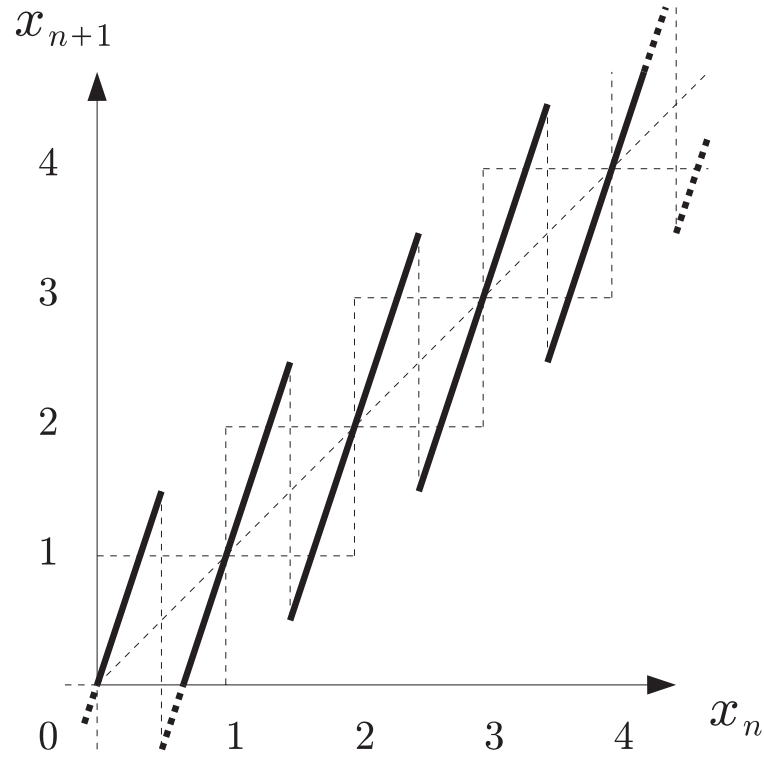

FIG. 1. Piecewise-linear map Eq. (23).

$$
\begin{aligned}
I_{q}(\omega)= & \int v^{(0)}(x)[u[x]-u(q)] \\
& \times\left[J_{q}(\omega)+J_{q}(-\omega)-1\right][u[x]-u(q)] h^{(0)}(x) d x,
\end{aligned}
$$

where $J_{q}(\omega)=1 /\left[1-\left(e^{i \omega} / \nu_{q}^{(0)}\right) \mathcal{H}_{q}\right], v^{(0)}(x)$ and $h^{(0)}(x)$ are, respectively, the left and right eigenfunctions corresponding to the maximum eigenvalue $\nu_{q}^{(0)}$ of $\mathcal{H}_{q}$.

\section{SIMPLE EXAMPLE OF DETERMINISTIC DIFFUSION}

It is usually difficult to obtain statistical structure functions by means of analysis. However, in simple systems such as piecewise linear maps, it is sometimes possible to analytically calculate such functions depending on the parameters involved in the systems. This section will introduce piecewise linear maps which present with chaotic diffusion and will set the parameters of these systems at the simplest value which allows Markov partition, with the goal of analytically deducing statistical structure functions such as a generalized diffusion coefficient $D_{q}$ and a generalized power spectrum $I_{q}(\omega)$. Other concrete examples of piecewise linear maps with Markov partition are analyzed in Refs. [13-15].

\section{A. Piecewise linear maps and chaotic diffusion}

Let us take an example of the following map:

$$
x_{n+1}=f\left(x_{n}\right)=\left\{\begin{array}{ll}
a\left(x_{n}-N\right)+N, & \left(N<x_{n} \leqslant N+\frac{1}{2}\right), \\
a\left(x_{n}-N-1\right)+N+1 & \left(N+\frac{1}{2}<x_{n} \leqslant N+1\right),
\end{array} \quad \text { for }{ }^{\forall} N \in \mathbb{N} .\right.
$$


This is a piecewise-linear map depicted by drawing two lines (with a gradient of $a$ ) from the lower left vertex to the upper right vertex within a $1 \times 1$ square and arranging them in a stepwise manner as shown in Fig. 1.

If parameter $a$ is set as $a>2$ and an appropriate initial point except for unstable periodic points is given to it, the time series $\left\{x_{n}\right\}$ will show chaotic motion, resembling random walks. If mapping is repeated, while placing several initial points close to each other, individual points will gradually part from each other, resembling diffusion.

Unlike ordinary diffusion which is induced by random factors such as thermal motion of molecules, etc., this map can show diffusive motion which is determined only by the mixing property of the chaotic map and not by any stochastic elements, if the initial point is fixed. Such diffusion is called chaotic diffusion; it is sometimes called deterministic diffusion on the grounds that subsequent motions are determined by the initial point in a deterministic manner [16-18].

Now, let us consider how we can obtain the generalized diffusion coefficient $D_{q}$ and the generalized power spectrum $I_{q}$ in this mapping system. In a simple piecewise-linear mapping system like this one, it is sometimes possible to obtain statistical structure functions analytically by setting the parameters of the system at levels which allow Markov partition. At one of the simplest values of the parameter allowing Markov partition, we will describe the method for statistically deducing $D_{q}$ and $I_{q}$, using the generalized FrobeniusPerron operator $H_{q}$.

\section{B. Markov partition of piecewise-linear maps and their expression using the matrix of the generalized Frobenius-Perron operator}

Regarding Eq. (23) shown in the preceding section, let us consider replacing the location $x_{n}$ of particles at time $n$ with $x_{n}=X_{n}+x_{n}^{\prime}$. Here $X_{n}$ and $x_{n}^{\prime}$ are, respectively, the integer and fractional part of $x_{n}$. Now, Eq. (23) can be rewritten as follows:

$$
\begin{gathered}
X_{n+1}=X_{n}+\Delta\left(x_{n}^{\prime}\right) \\
x_{n+1}^{\prime}=g\left(x_{n}^{\prime}\right) \\
\Delta\left(x_{n}^{\prime}\right)=\left\lfloor f\left(x_{n}^{\prime}\right)\right\rfloor \\
g\left(x_{n}^{\prime}\right)=f\left(x_{n}^{\prime}\right)-\left\lfloor f\left(x_{n}^{\prime}\right)\right\rfloor\left(X_{n} \in \mathbb{N}, 0<x_{n}^{\prime} \leqslant 1\right) .
\end{gathered}
$$

The map $g$ defined by Eq. (24) is a chaotic map. For almost all the initial points, the trajectories become chaotic.

The motion of $X_{n}$ can be viewed as random walk on the one-dimensional lattice, with the lattice point $\Delta\left(x_{n}^{\prime}\right)$ of each unit interval serving as a jump. In Eq. (24), the time series $\left\{X_{n}\right\}$ is dependent only on $\left\{x_{n}^{\prime}\right\}$. In other words, $\left\{X_{n}\right\}$ is generated from the map $g$. As described in the previous section, the diffusion coefficient $D_{q}$ of $X_{n}$ 's motion can be obtained if the generalized Frobenius-Perron operator $H_{q}$ for $g$ is given.

In the case of $a=1+\sqrt{3}$, the map $g$ can be subjected to the Markov partition. Let us divide the unit interval $[0,1]$ into four subintervals $p_{1}=[0,1 /(1+\sqrt{3})], p_{2}=[1 /(1+\sqrt{3}), 1 / 2]$, $p_{3}=[1 / 2,1-1 /(1+\sqrt{3})]$, and $p_{4}=[1-1 /(1+\sqrt{3}), 1]$. The subintervals $p_{1}$ and $p_{4}$ are uniformly mapped to the whole unit interval $p_{1} \cup p_{2} \cup p_{3} \cup p_{4}=[0,1]=g\left(p_{1}\right)=g\left(p_{4}\right) ; p_{2}$ and $p_{3}$ to $p_{1}=g\left(p_{2}\right)$ and $p_{4}=g\left(p_{3}\right)$, respectively. Therefore if this map is subjected to time evolution, with the initial distribution being uniform, the probability distribution at all times is constant within each subinterval. This means that in maps which can be subjected to Markov partition, the generalized Frobenius-Perron operator of the map can be expressed in the form of a matrix. The generalized Frobenius-Perron operator, $H_{q}(a)$, can usually be expressed by the following matrix if $a=1+\sqrt{3}$.

$$
\begin{aligned}
H_{q}(1+\sqrt{3}) & =\frac{1}{1+\sqrt{3}}\left(\begin{array}{cccc}
1 & 1 & 0 & 1 \\
1 & 0 & 0 & 1 \\
1 & 0 & 0 & 1 \\
1 & 0 & 1 & 1
\end{array}\right) \\
& \times\left(\begin{array}{cccc}
e^{q 0} & 0 & 0 & 0 \\
0 & e^{q 1} & 0 & 0 \\
0 & 0 & e^{q(-1)} & 0 \\
0 & 0 & 0 & e^{q 0}
\end{array}\right) \\
= & \frac{1}{1+\sqrt{3}}\left(\begin{array}{cccc}
1 & e^{q} & 0 & 1 \\
1 & 0 & 0 & 1 \\
1 & 0 & 0 & 1 \\
1 & 0 & e^{-q} & 1
\end{array}\right) .
\end{aligned}
$$

Of the matrices contained in Eq. (25), the left matrix indicates the connections of each subinterval. If $p_{i}$ is mapped into cell $p_{j}$, the row $j$ line $i$ component of this matrix is defined as 1 . If $p_{i}$ is not mapped into $p_{j}$, this component is defined as 0 . In the right matrix, the $i$ th diagonal component is $\exp \left(q \Delta_{i}\right)\left(\Delta_{i}\right.$ indicates the value of $\Delta$ corresponding to $\left.p_{i}\right)$ and is equivalent to $e^{q u[x]}$ of Eq. (20).

Klages and Dorfman imposed periodic boundary conditions on their Markov process. In other words, they truncated infinite dimensional transition matrices to obtain conventional diffusion coefficients [3]. In contrast, we introduced the integer and fractional parts of dynamics in Eq. (24), so that we have only to solve eigenvalue problems of finite dimensional generalized Frobenius-Perron matrices $H_{q}$. This is based on the fact that (generalized) diffusion coefficients are determined by the correlation functions of the velocity, namely, the fractional part of the dynamics only. Thus we can obtain generalized diffusion coefficients including conventional ones more precisely without using matrix truncation.

\section{Deduction of statistical structure functions and $D_{q}(a)$ and $I_{q}(a, \omega)$ when $a=1+\sqrt{3}$}

The maximum eigenvalue $\nu_{q}^{(0)}(a)$ of matrix $H_{q}(a)$ when $a=1+\sqrt{3}$ can be calculated as follows: 


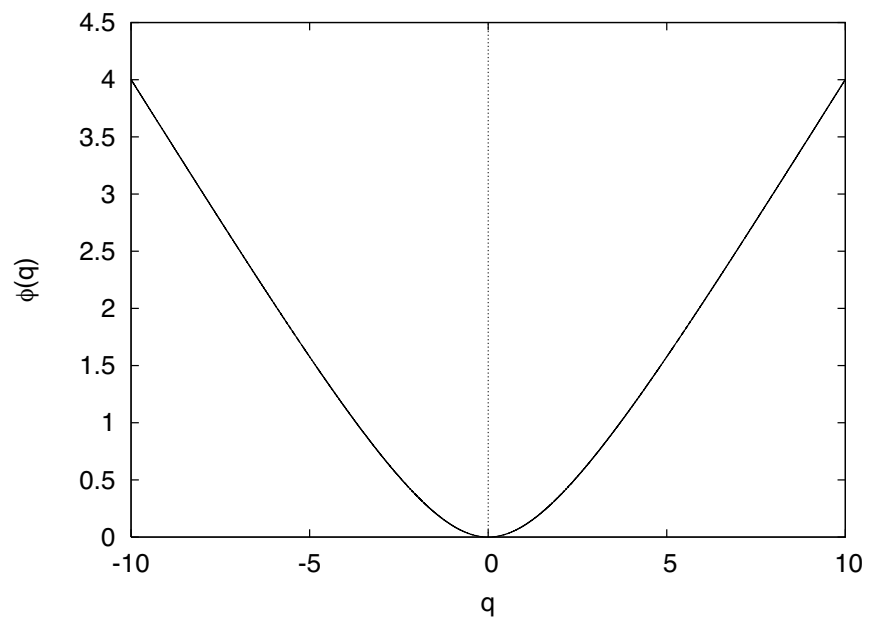

FIG. 2. Characteristic function $\phi(q)(a=1+\sqrt{3})$.

$$
\nu_{q}^{(0)}(1+\sqrt{3})=\frac{e^{q}+\sqrt{e^{q}\left(e^{2 q}+e^{q}+1\right)}}{(1+\sqrt{3}) e^{q}}
$$

From this value, we can deduce the statistical structure functions for the time series of the velocity of the chaotic diffusion $\left\{\Delta\left(x_{n}^{\prime}\right)\right\}$ as follows.

$\left.\phi(q)\right|_{a=1+\sqrt{3}}=\log \nu_{q}^{(0)}(1+\sqrt{3})=\log \frac{A_{1}+A_{2}}{(1+\sqrt{3}) A_{1}}$,

$$
\left.u(q)\right|_{a=1+\sqrt{3}}=\left.\phi^{\prime}(q)\right|_{a=1+\sqrt{3}}=\frac{A_{1}\left(A_{1}^{2}-1\right)}{2 A_{2} A_{3}},
$$

$$
\begin{aligned}
D_{q}(1+\sqrt{3})= & \frac{A_{1}^{3}}{8 A_{2}^{3} A_{3}^{2}}\left(A_{1}^{4}+2 A_{1}^{3}+2 A_{1}^{2} A_{2}+6 A_{1}^{2}+8 A_{1} A_{2}+2 A_{1}\right. \\
& \left.+2 A_{2}+1\right)
\end{aligned}
$$

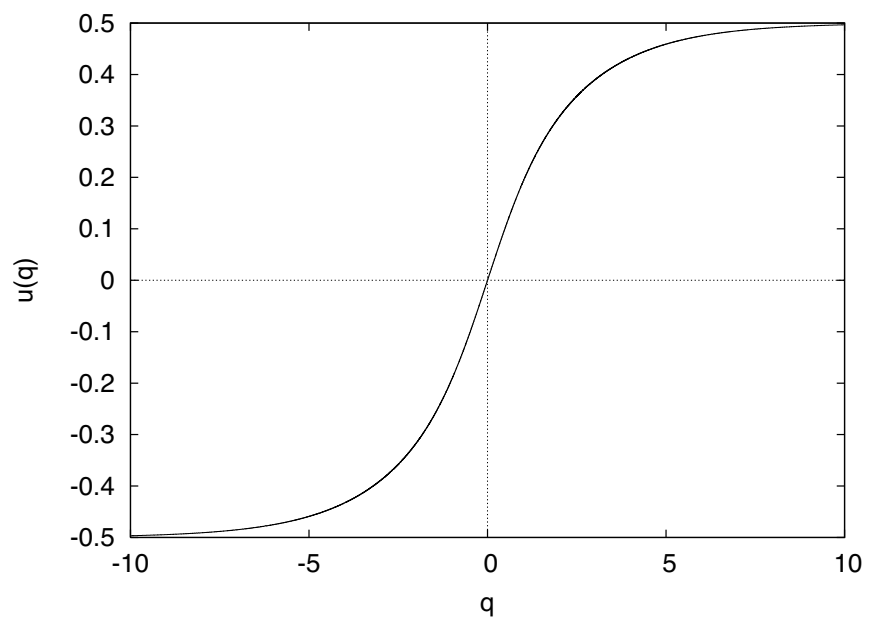

FIG. 3. Weighted velocity average $u(q)(a=1+\sqrt{3})$.

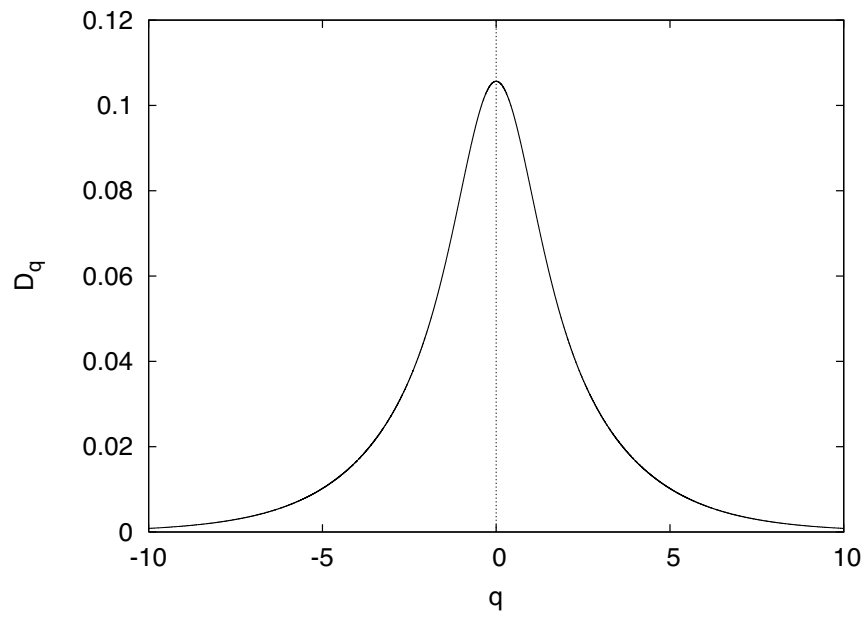

FIG. 4. Generalized diffusion coefficient $D_{q}(1+\sqrt{3})$.

$$
\begin{aligned}
I_{q}(1+\sqrt{3}, \omega)= & \frac{B_{1} \cos \omega+B_{2}}{B_{3} \cos \omega+B_{4}} \\
& \times \frac{\left(A_{1}-A_{2}\right)\left(A_{1}^{3}-A_{2}\right)}{2 A_{1}\left(A_{1}^{2}-A_{1}^{2} A_{2}+A_{1}+1\right)\left(A_{1}^{2}+1\right)},
\end{aligned}
$$

where

$$
\begin{gathered}
A_{1}=e^{q}, \\
A_{2}=\sqrt{e^{q}\left(e^{2 q}+e^{q}+1\right)}, \\
A_{3}=e^{q}+\sqrt{e^{q}\left(e^{2 q}+e^{q}+1\right)}, \\
B_{1}=4 A_{1}^{4}+8 A_{1}^{3}+8 A_{1}^{2} A_{2}+4 A_{1}^{2}, \\
B_{2}=3 A_{1}^{5}+A_{1}^{4} A_{2}+8 A_{1}^{4}+4 A_{1}^{3} A_{2}+10 A_{1}^{3}+6 A_{1}^{2} A_{2}+8 A_{1}^{2} \\
+4 A_{1} A_{2}+3 A_{1}+A_{2}, \\
B_{3}=A_{1}^{4}+2 A_{1}^{3}+2 A_{1}^{2} A_{2}+2 A_{1}^{2}+2 A_{1}+2 A_{2}+1, \\
B_{4}=A_{1}^{4}+4 A_{1}^{3}+2 A_{1}^{2} A_{2}+6 A_{1}^{2}+4 A_{1} A_{2}+4 A_{1}+2 A_{2}+1 .
\end{gathered}
$$

These equations are graphically represented in Figs. 2-5.

The $u(q)$ shown in Fig. 3 is a weighted average of the time series of the velocity of the chaotic diffusion $\left\{\Delta\left(x_{n}^{\prime}\right)\right\}$. According to the framework of the theory of large deviation statistics, $u$ at $q=0$, i.e., $u(q=0)$, should be equal to 0 , identical to the ordinary average $\left\{\Delta\left(x_{n}^{\prime}\right)\right\}$ due to the symmetry of map $g$. In fact, $u(0)$ is equal to 0 in the graph. In the same way, $D_{0}(1+\sqrt{3})$ at a $q$ value of 0 is identical to the diffusion coefficient $D$ (Fig. 4), and the $I_{0}(1+\sqrt{3}, \omega)$ in Fig. 5 is equal to the conventional power spectrum obtained by the ordinary method, without involving weighting. If $q$ is increased from 0 to $\infty$, the weighted average $u(q)$ increases, eventually approaching $1 / 2$. That is, of the various processes involved in chaotic diffusion, the $q$ corresponding to the process with largest fluctuation is equal to $\infty$, and the average velocity 


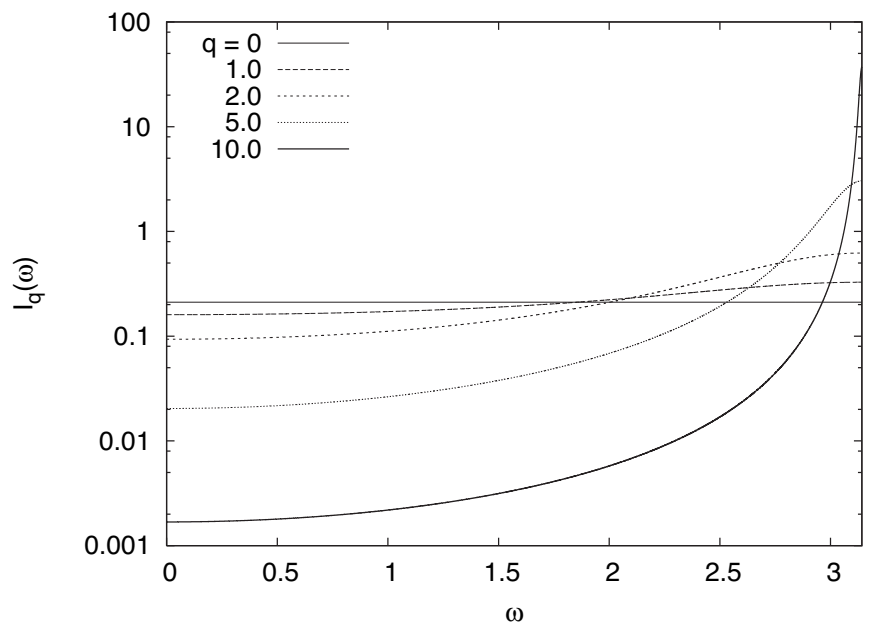

FIG. 5. Generalized power spectrum $I_{q}(1+\sqrt{3}, \omega)$.

at that time is $1 / 2$. In the same way, the generalized diffusion coefficient $D_{q}$ also approaches the diffusion coefficient $D_{\infty}(1+\sqrt{3})=0$, which corresponds to the process with largest fluctuation, as $q$ increases to $\infty$. The generalized power spectrum $I_{q}(1+\sqrt{3}, \omega)$ approaches the delta function $I_{\infty}(1+\sqrt{3}, \omega)=\delta(\omega-\pi)$ as $q$ increases to $\infty$. If we consider that the average of $\Delta\left(x_{n}^{\prime}\right)$ is $1 / 2$, the diffusion coefficient is 0 and the power spectrum serves as a delta function, we may say that the orbit with the greatest fluctuation when $a=1$ $+\sqrt{3}$ is the drift orbit (Fig. 6) occurring from the period two orbits of $x_{n}^{\prime}$ shown in Fig. 7.

\section{NUMERICAL CALCULATION OF GENERALIZED DIFFUSION COEFFICIENT $D_{q}(a)$ AND GENERALIZED POWER SPECTRUM $I_{q}(a, \omega)$ AND ITS RESULTS}

It is known that in the chaotic diffusion system represented by Eq. (23) there is a complex dependence of diffusion coefficient $D(a)$ on parameter $a$. Our numerical calculation revealed that the generalized diffusion coefficient $D_{q}(a)$ and the generalized power spectrum $I_{q}(a, \omega)$ are also dependent on the parameter in a complex manner. It was additionally found that, among the various possible orbits for this system, the drift orbit shows a relationship between parameter $a$ and average velocity that can be graphically represented in a form resembling the devil's staircase. This section will present these findings, using graphic representations.

\section{A. Numerical calculation of generalized diffusion coefficient $D_{q}$ and its results}

Klages and Dorfman discovered that an irregular relationship is present between parameter $a$ and diffusion coefficient $D(a)$ in the chaotic diffusion system defined by Eq. (23) [3]. They called the irregular parameter dependence of the diffusion coefficient a "fractal diffusion coefficient" on the grounds that more minute structures continue to appear within the irregular curve when a part of the curve is magnified with progressively higher powers $[3,19-21]$. This

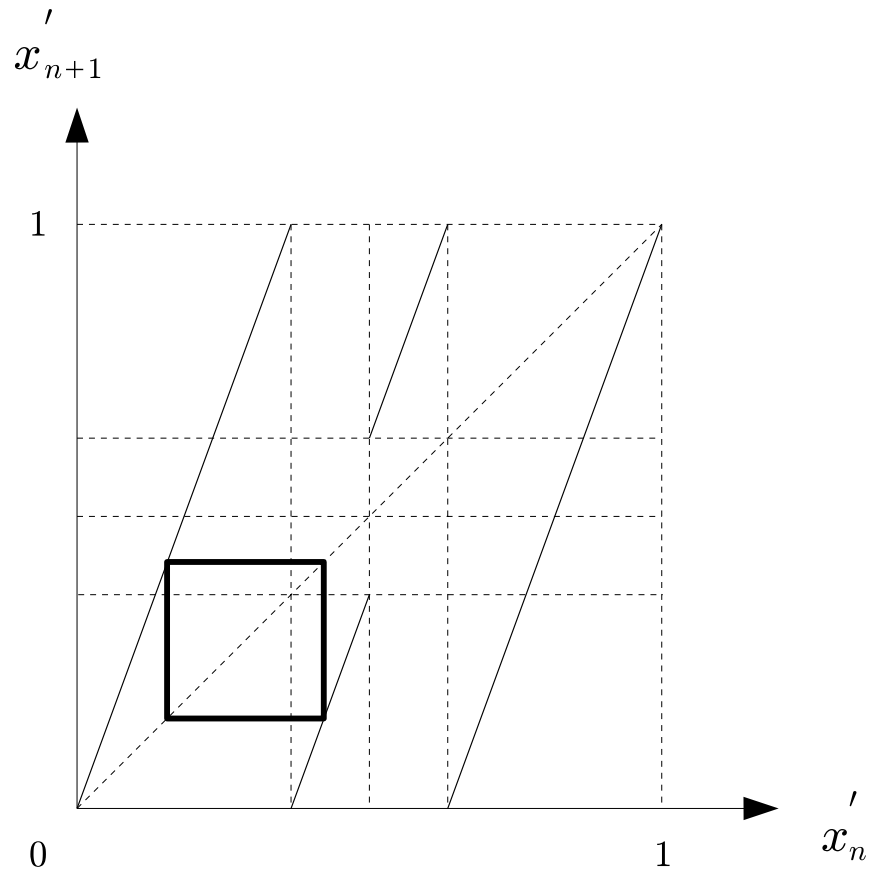

FIG. 6. Period-two trajectory in the map $g$ giving dynamics of the fractional part of the original ones.

curve represents an ordinary diffusion coefficient for this system. What curve will be depicted by the generalized diffusion coefficient, i.e., the diffusion coefficient expanded within the framework of the theory of large deviation statistics? To obtain this curve, we should first obtain values of $a$ (for which a Markov partition is possible) in a number large enough to fill the $a$ axis adequately, according to the method described in Refs. $[19,20]$. For each value of $a$ thus obtained, we then calculate the maximum eigenvalue $\nu_{q}^{(0)}(a)$ of the generalized Frobenius-Perron operator $H_{q}(a)$ for the map $g$ discussed in the preceding chapter. Then, the equation $D_{q}(a)=1 / 2\left(\partial^{2} / \partial q^{2}\right) \log \nu_{q}^{(0)}(a)$ is used. In this way, the curve shown in Fig. 8 can be obtained.

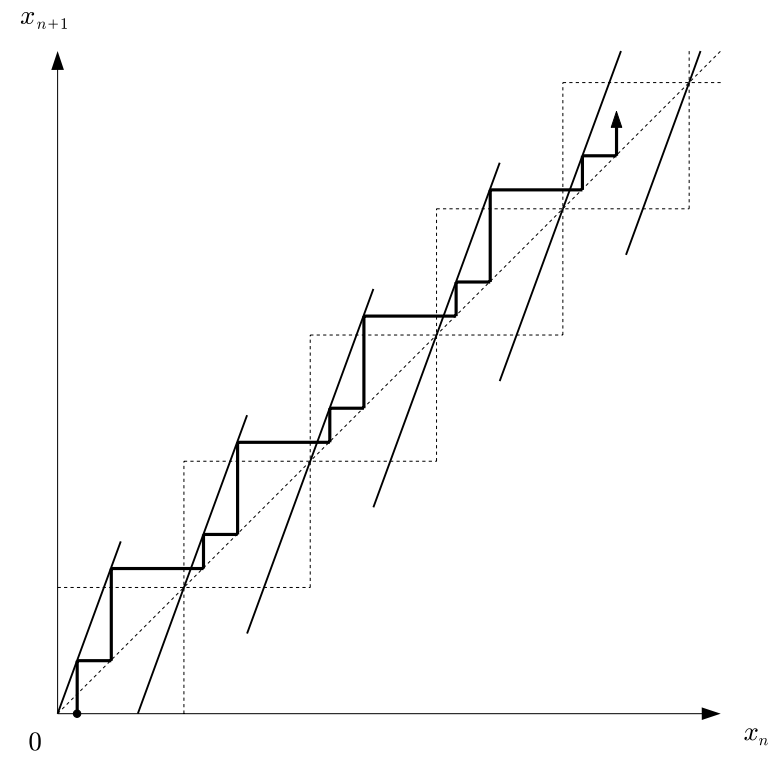

FIG. 7. Period-two drift motion of the original dynamics. 


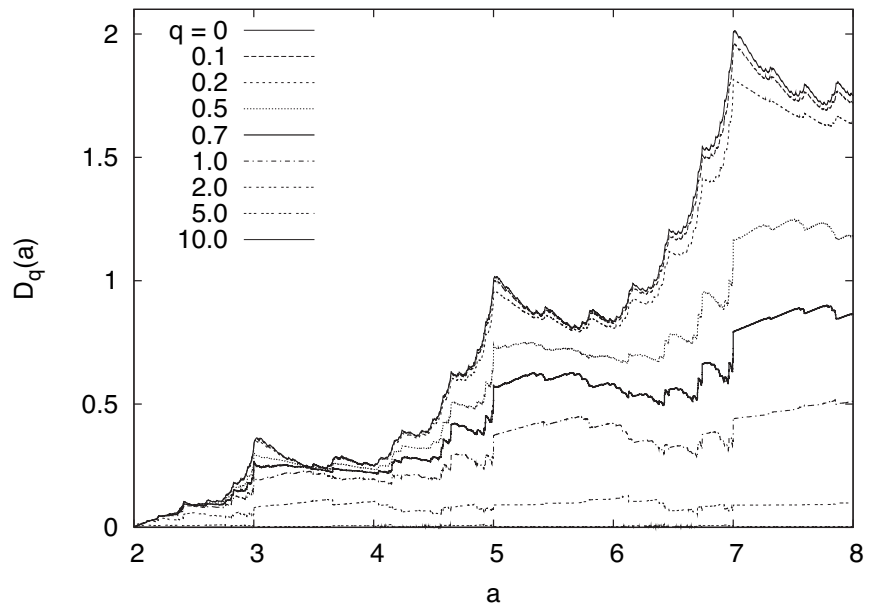

FIG. 8. Parameter $a$ dependence of the generalized diffusion coefficient $D_{q}(a)$. About 5500 single data points are connected by a line segment for a fixed value of $q$.

As $q$ is gradually increased from 0 , the curve descends, eventually converging at 0 when $q$ approaches $\infty$. During this process, it appears that the peaks of the curve are gradually smoothed, resulting in a gradual increase in the percentage of the relatively flat parts in the entire curve. On the basis of this finding, we estimate that if the fractal dimension $d_{f}(q)$ for the curve $D_{q}(a)$ for the interval $2 \leqslant a \leqslant 8$ is measured, it will gradually decrease from its value at $D_{0}(a)$, eventually approaching 1. Figure 9 shows the results of measuring the fractal dimension $d_{f}(q)$ of curve $D_{q}(a)$ at varying values of $q$, using the box counting method [22].

Now we discuss the results of $d_{f}(q)$ measurement. Regarding the fractal dimension of $D_{0}(a)$, we obtained $d_{f}(0)$ $\simeq 1.046$. This indicates that $d_{f}(q)$ shows a monotonic decrease until $q=0.2$. If $q$ is larger than $0.2, d_{f}(q)$ depicts an upward convex curve. We think that this local minimum is smooth and cannot account for this minimization. Maximum $d_{f}\left(q_{\max }\right) \simeq 1.05$ was recorded when $q_{\max }$ was 0.6 to 0.7 . In Fig. 8, the curve for $q=0.7$ is shown as a solid line. The curve shown with this solid line can be viewed as having the

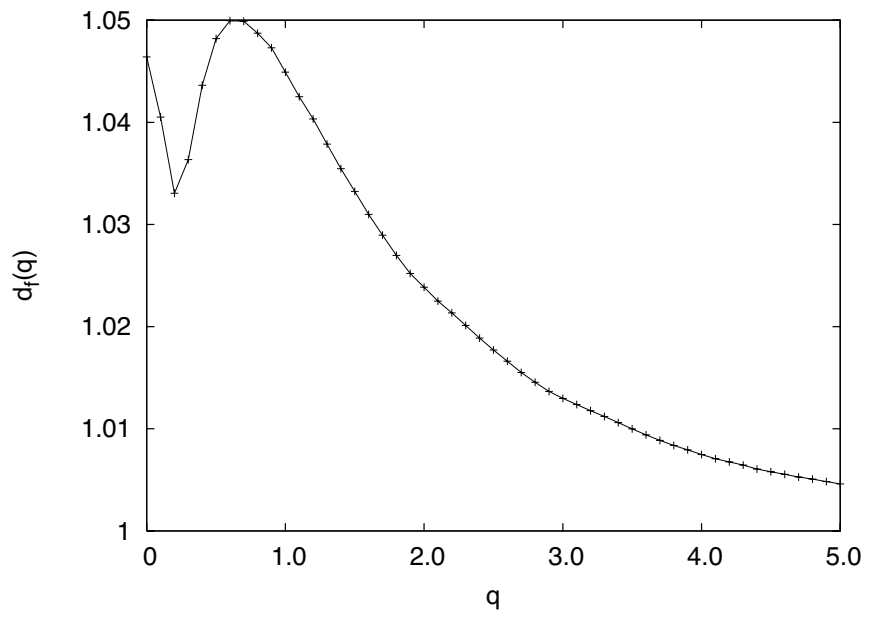

FIG. 9. Weight index $q$ dependence of the fractal dimension $d_{f}(q)$ of the curves $D_{q}(a)$ plotted against $a$.
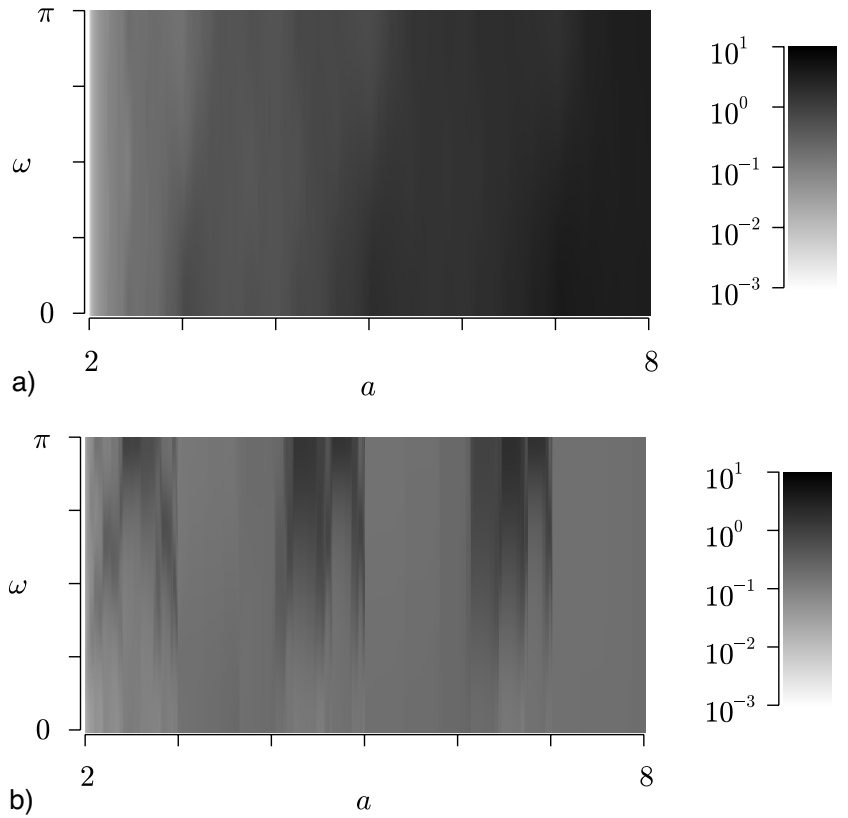

b)
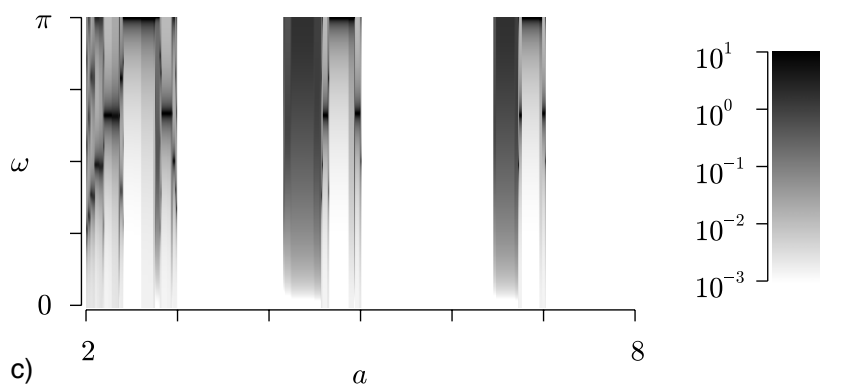

FIG. 10. Generalized power spectra $I_{q}(a, \omega)$ for $q=0$ (a), 2 (b), and 10 (c). The number of points is equal to 600 in the $a$ direction, 314 in the $\omega$ direction.

highest fractal dimension among $D_{q}(a)$. As illustrated above, not only the diffusion coefficient $D(a)$ but also the generalized diffusion coefficient $D_{q}(a)$ assumed a complex form in the chaotic diffusion system defined by Eq. (23).

\section{B. Numerical calculation of generalized power spectra $I_{q}(a, \omega)$ and its results}

As described in Sec. III C, the generalized power spectrum $I_{q}(a, \omega)$ allowing a Markov partition can be obtained by calculating $H_{q}(a)$ and its maximum eigenvalue $\nu_{q}^{(0)}(a)$ and the corresponding right and left eigenvectors and by applying these values to Eq. (22). Because $I_{q}(a, \omega)$ involves three variables $(q, a, \omega)$, the results of calculation need to be expressed on the $a-\omega$ plane for full characterization of $I_{q}(a, \omega)$. Figure 10 shows the results of numerical calculation at $q$ $=0,1,2,5$, and 10 in images. In these images, the horizontal direction indicates $a$ and the vertical direction corresponds to $\omega$. The values of $I_{q}(a, \omega)$ at a given point are presented on the plane in gray.

Parameter $a$ dependence of $I_{q}(a, \omega)$ for $\omega=0$ and $\pi$ is shown below. It is noticeable from these figures that the features of $I_{q}(a, \omega)$ differ greatly between the case of $q=0$ and 

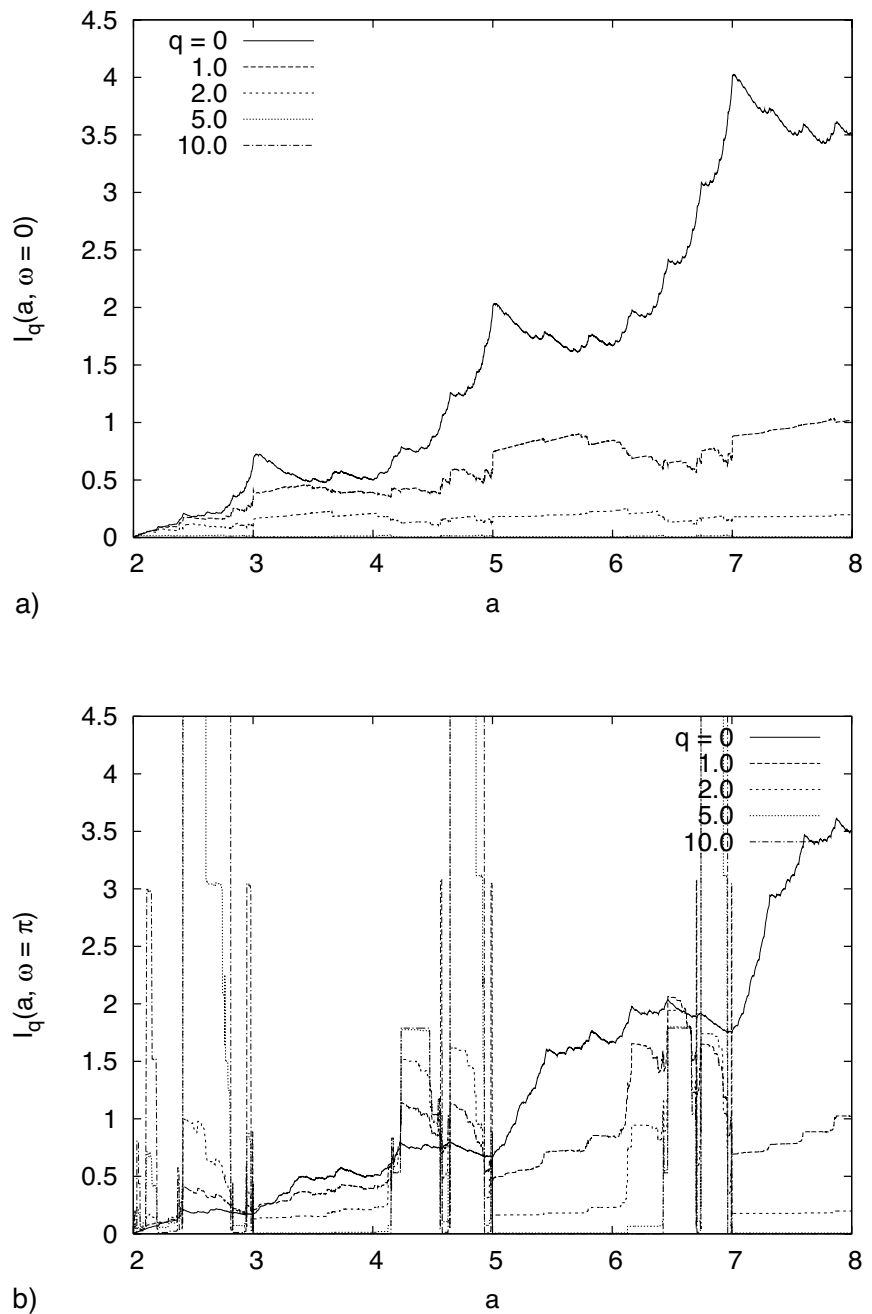

FIG. 11. Parameter $a$ dependence of the generalized power spectra $I_{q}(a, \omega)$ for $\omega=0$ (a) and $\pi$ (b). About 5500 single data points are connected by a line segment for a fixed value of $q$.

the case of larger $q$ (particularly the case when $q=10$ ). In Fig. 10 , the plane $I_{0}(a, \omega)$ at $q=0$ shows little change in the vertical direction, while it assumes higher values in the vertical direction as $a$ increases. However, this increase is not a monotonic increase but is really a repetition of small increases depending on the parameter as shown in Fig. 11. However, when $q=10$ (Fig. 10), a region with relatively high values and a region with small values appear in an alternating manner in the horizontal direction. In the areas with large
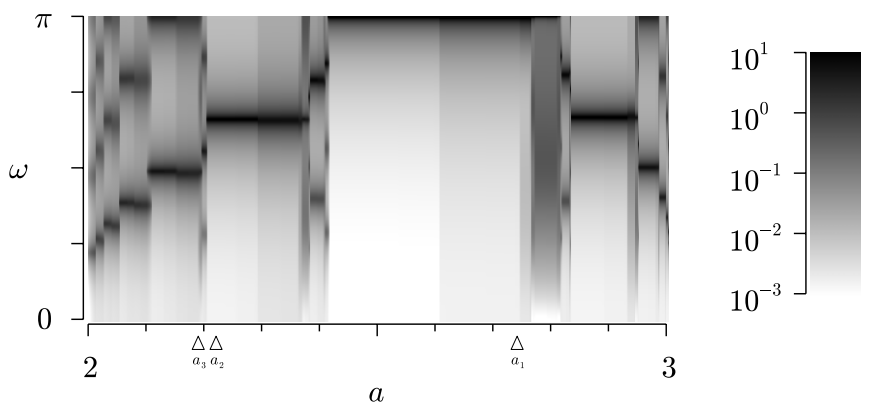

FIG. 12. Generalized power spectra $I_{q=10}(a, \omega)$ for $2 \leqslant a \leqslant 3$. values in this case, the maximum value remains almost constant irrespective of the increase in $a$. Let us examine the region $2 \leqslant a \leqslant 3$ of the plane $I_{q}(a, \omega)$ when $q=10$ (Fig. 10) in more detail. Figure 12 is a magnification of the region 2 $\leqslant a \leqslant 3$ of Fig. 10 .

In this region, the plane $I_{q}(a, \omega)$ is a curve possessing one or multiple peaks in the locations satisfying $\omega$ $=\left(l_{a} / m_{a}\right) \pi\left(l_{a}, m_{a} \in \mathbb{Z}, l_{a} \leqslant m_{a}\right)$ when $a$ is fixed at a certain value. In Sec. III C, we calculated the generalized power spectrum for $a=1+\sqrt{3}\left(a_{1}\right.$ in Fig. 12) in this area. Numerical calculation revealed that a sharp peak appears in the vicinity of $\omega=\pi$ when $q=10$ (Fig. 5). In fact, when the $a_{1}$ in Fig. 12 is checked, the area in the vicinity of $\omega=\pi$ is black. When $q \rightarrow \infty$, this spectrum becomes a delta function which has values only at $\omega=\pi[=(1 / 2) 2 \pi]$, and the corresponding $x_{n}$ time series was found to correspond to period-two drift orbits. The same can be said of $a$ other than $a=a_{1}$. That is, the black gradational peak in the locations satisfying $\omega$ $=\left(l_{a} / m_{a}\right) \pi=\left(l_{a} / 2 m_{a}\right) 2 \pi$ loses the intermediate gray values and becomes a black horizontal line when $q \rightarrow \infty$. As a result, the corresponding $x_{n}$ motion may become a drift orbit with a period of $2 m_{a} / l_{a}$. For example, in the cases of $a_{2}$ $=2.1903 \ldots, a_{3}=2.2207 \ldots$ (Fig. 12), each spectrum has a peak at $\omega=2 \pi / 3,2 \pi / 4\left[I_{q=10}\left(a_{3}, \omega\right)\right.$ in Fig. 13 peaks not only at $2 \pi / 4$ but also at $2 \pi / 2]$, and the weighted averages of the orbit's velocity $u(q)$ converge at $1 / 3$ and $1 / 4$, respectively, when $q \rightarrow \infty$ (Fig. 13). When $2<a \leqslant 4$, it is evident that $\Delta\left(x_{n}^{\prime}\right)$ assumed only three values $(0,1,-1)$, in view of the shape of its map. Therefore the time series of the drift orbit's velocity when $a=a_{2}, a_{3}$ and $q \rightarrow \infty$ will be defined as

$$
\begin{aligned}
& \left\{\Delta\left(x^{\prime}{ }_{n}\right)\right\}=\{\ldots, 0,0,1,0,0,1,0,0,1,0,0,1, \ldots\}\left(a=a_{2}\right), \\
& \left\{\Delta\left(x^{\prime}{ }_{n}\right)\right\}=\{\ldots, 0,0,0,1,0,0,0,1,0,0,0,1, \ldots\}\left(a=a_{3}\right) .
\end{aligned}
$$

This area contains other spectra corresponding to drift orbits of varying periods of cycle, and the location of their peak is dependent on $a$ in a complex manner. In Fig. 10, areas with a peak in the vertical direction are visible not only in the vicinity of $2<a \leqslant 3$ but also in the vicinity of 4.55 $\leqslant a \leqslant 5$ and $6.7 \leqslant a \leqslant 7$. These areas are the same in structure to the area $2 \leqslant a \leqslant 3$, except for the fact that they have been reduced in size in the horizontal direction.

\section{Complex dependence of $u(q)$ on $a$}

Some areas in Fig. 10 seem to be white, since the generalized power spectrum approaches a delta function located at the angular frequency corresponding to the period of the unstable periodic points causing the ballistic motion. For instance, in the seemingly white area in the vicinity of $a=1$ $+\sqrt{3}$ the generalized power spectrum $I_{q}(\omega)$ is given by $\delta(\omega$ $-\pi)$ in the limit of $q \rightarrow \infty$. The angular frequency $\omega=\pi$ corresponds to the period- 2 unstable periodic points shown in Fig. 6, which also yield a ballistic motion shown in Fig. 7. In other seemingly white areas, $I_{\infty}(\omega)$ are found to be delta functions in the same way.

To examine this in detail, let us see how the weighted average of the orbit's velocity $u(q)$ will converge when $q$ 


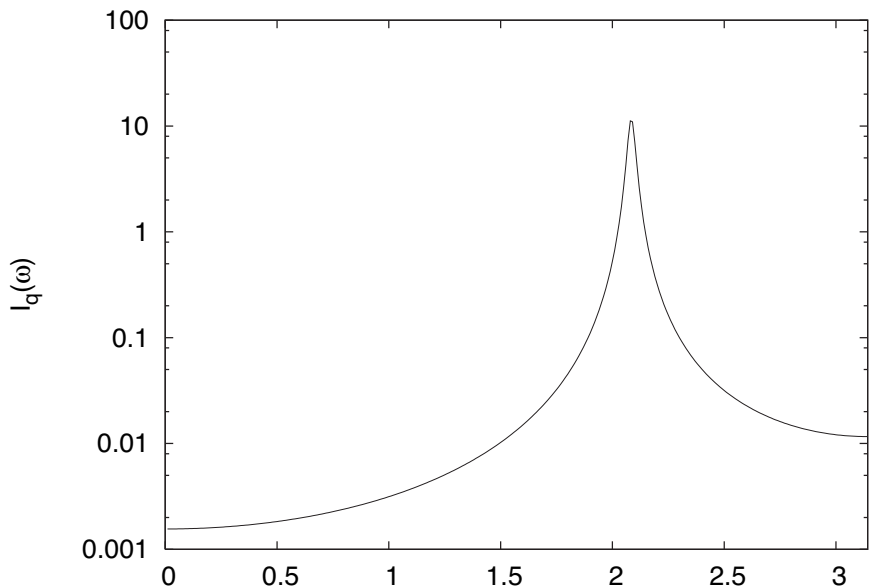

a)

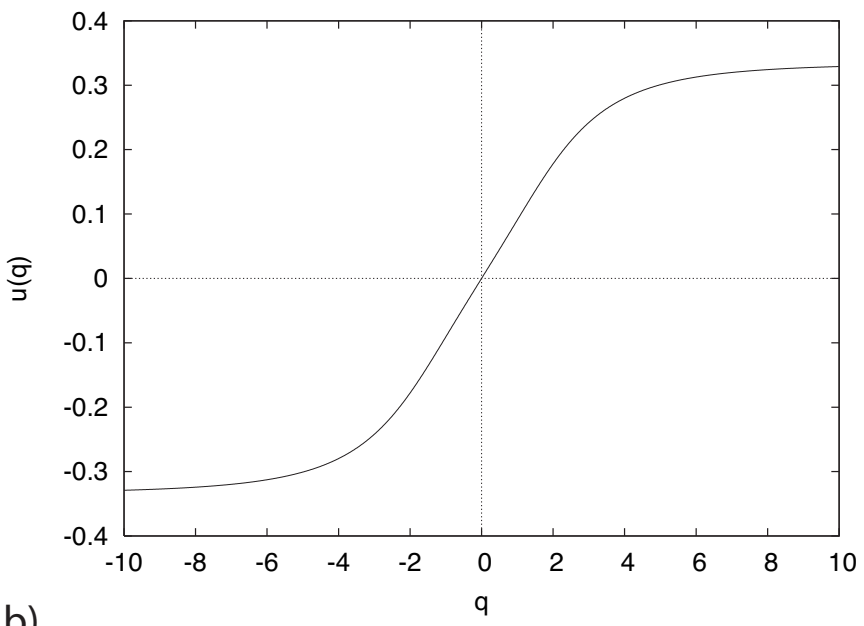

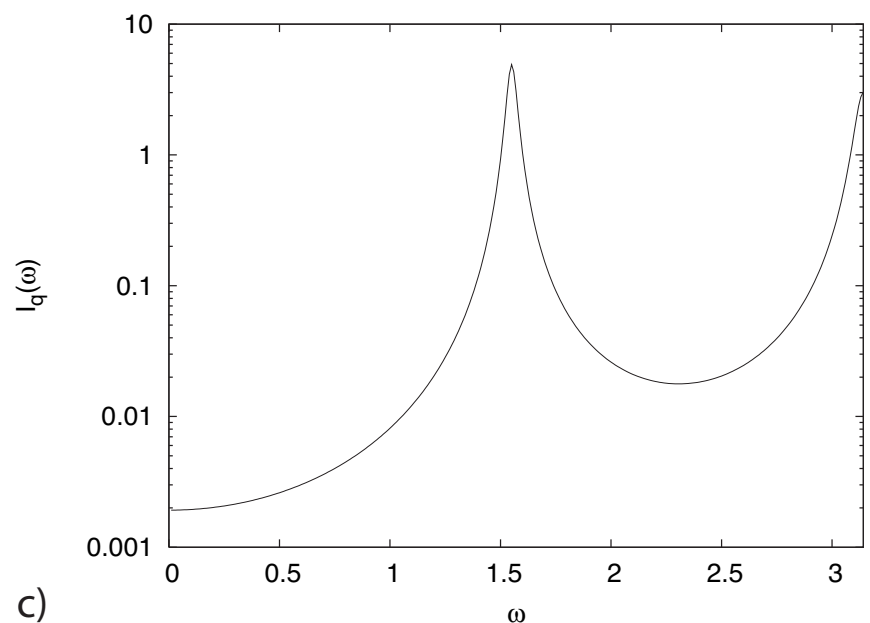

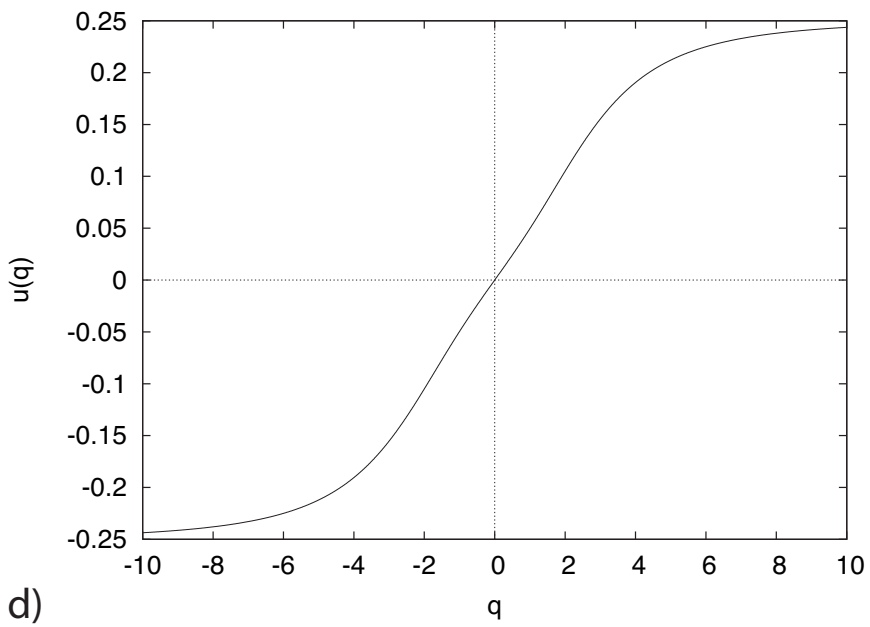

FIG. 13. Generalized power spectrum $I_{q=10}(a, \omega)$ and weighted average velocity $u(q)$ at $a=a_{2}=2.1903 \ldots$ (a),(c) and $a=a_{3}=2.207 \ldots$ (b) (d).

$\rightarrow \infty$. Figure 14 graphically represents the relationship between parameter $a$ and $u(q)$ when $q=0-10$. Here, the partial differential related to $q$ of $u(q)$ is $2 D_{q}(a)$, according to Eq. (13). The curve of $D_{q}(a)$ (Fig. 8) shows that $D_{10}(a) \simeq 0$. We may therefore say that the graph of $a, u(10)$ at $q=10$ (solid line in the graph) adequately converges to the graph of $a$, $u(\infty)$ at $q=\infty$. It seems therefore rational to deem the $q=10$ graph as $q=\infty$. The $q=10$ graph depicted with this solid line resembles the graph of a function called the "devil's staircase." Most of the graphs presenting the relationship between parameter $a$ and the mean velocity of the drift orbit possessed by the corresponding mapping system (the mapping system with the highest velocity) have a gradient of 0 at most values of $a$. But their gradient shows a monotonic increase as $a$ increases when the entire graph is viewed.

In this graph, the value of $u(q \rightarrow \infty)$ when $3<a<4.2$ is 1 . When $2<a \leqslant 4$, the value of $\Delta\left(x_{n}^{\prime}\right)$ is either 0,1 , or -1 . Therefore at least in the range of $3<a \leqslant 4$, the time series of the corresponding drift orbit's velocity assumes the following constant value:

$$
\left\{\Delta\left(x^{\prime}{ }_{n}\right)\right\}=\{\ldots, 1,1,1,1,1,1,1,1,1,1,1,1, \ldots\} .
$$

That is, since the period of the time series of the velocity is unity, the power spectrum of this time series, $I_{\infty}(a, \omega)$, has delta function values only when $\omega=2 \pi / 1 \bmod 2 \pi=0$.

In the above-mentioned range $3<a<4.2$, an unstable fixed point is formed somewhere other than the point 0,1 of the map $g$, and this point is 1 in the map $\Delta$. Furthermore, in this range, the mean velocity of the fastest drift orbit, $u(q)$, assumes a value of 1 . If the initial point is located immediately above this unstable fixed point under these conditions, the time series of $\Delta\left(x_{n}^{\prime}\right)$ is 1 at all values of $n$. As a result, the power spectrum will become visible for the reasons mentioned above. The white appearance of the range $5<a$ $\leqslant 6.5$ can be explained by similar reasoning. In this case, the time series of $\Delta\left(x_{n}^{\prime}\right)$ always assumes the value 2 .

\section{CONCLUDING REMARKS}

This paper dealt with extracting the non-Gaussian characteristics of the phenomenon of diffusion. We referred to the fact that the diffusion coefficient can represent only Gaussian characteristics of diffusion, and discussed the generalization of the diffusion coefficient and power spectrum within the framework of the theory of large deviation statistics, with the 


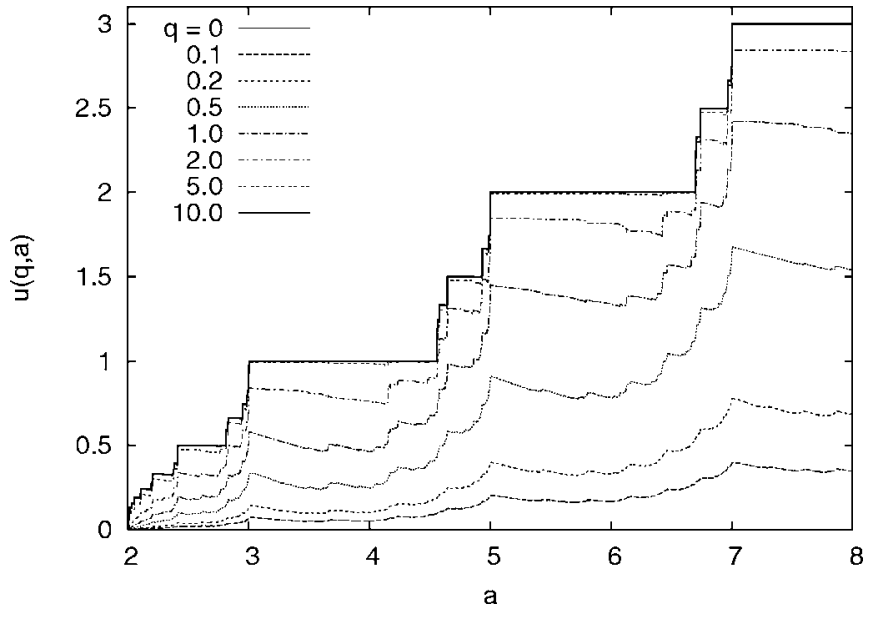

FIG. 14. Weighted velocity average $u(q, a)$ as a function of the parameter $a$ for $q=0,0.1,0.2,0.5,1.0,2.0,5.0$, and 10. About 5500 single data points are connected by a line segment for a fixed value of $q$.

goal of obtaining means for identifying both Gaussian and non-Gaussian characteristics. Then we referred to the mapping system in which Klages and Dorfman discovered a complex dependence of diffusion coefficients on the parameter. We attempted to extract the non-Gaussian characteristics of this system by calculating the generalized diffusion coefficient $D_{q}$ and the generalized power spectrum $I_{q}(\omega)$. The following results were obtained.

(1) The parameter of the system was set at $a=1+\sqrt{3}$, which is one of the simplest values allowing Markov partition. The statistical structure functions at that time, i.e., $\phi(q)$, $u(q)$ and $D_{q}(a), I_{q}(a, \omega)$, were obtained analytically. When the velocity was set at the temporal coarse graining level, the motion corresponding to the physical process with largest deviation from the average was identified as the period two drift motion (Fig. 7).

(2) The curve representing the relationship between parameter $a$ and $D_{q}(a)$ was numerically obtained for multiple $q$ values (Fig. 8). This revealed that an increase in $q$ leads to the appearance of flat parts in the curve. We then attempted to evaluate the complexity of the curve quantitatively by obtaining the fractal dimension $d_{f}(q)$ for these $D_{q}(a)$ curves (Fig. 8).

(3) The two-dimensional field for a given value of $q$, i.e., $I_{q}(a, \omega)$, was numerically obtained (Fig. 10). This disclosed that the motion corresponding to $q=\infty$ in this mapping system is a drift motion. It was also found that its period was dependent on parameter $a$ in a complex manner.

(4) A graph plotting the weighted mean velocity $u(q)$ of diffusive particles against parameter $a$ was numerically obtained (Fig. 14). This demonstrated that the graph $u(q \rightarrow \infty)$ corresponding to the average velocity of the drift orbit has a structure resembling the devil's staircase.

(5) It was found that the unstable periodic orbit of map $g$ corresponding to the drift motion with the highest velocity in this system changes in a complex manner depending on the value of parameter $a$. This seems to be one of the factors explaining why the graphs of $D_{q}(a)$ and $u(q)$ have complex structures.
The above-listed non-Gaussian characteristics of the chaotic diffusion system can be identified only within the framework of the theory of large deviation statistics. In this sense, we may say that the usefulness of the theory of large deviation statistics has been endorsed by this study.

Our results for fractal dimensions of the ordinary diffusion coefficient $D(a)$ of this model $d_{f}(0)$ agree with those obtained in Ref. [23]. However, the author of Ref. [24] argues that these noninteger dimensions are numerical artifacts of the logarithmic corrections. We think that the boxcounting method cannot distinguish between noninteger dimensions and logarithmic corrections. We feel intuitively that the irregularity of the parameter $a$ dependence of the generalized diffusion coefficient $D_{q}(a)$ shown in Fig. 8 decreases as the weight index $q$ increases. The weight index $q$ dependence of the fractal dimension $d_{f}(q)$ shown in Fig. 9 is, however, not monotonous. It is desirable to propose suitable quantities other than fractal dimensions which measure the irregularity of the parameter dependence of the conventional and generalized diffusion coefficients.

We mention here some related studies. In Refs. [3,19,20], so-called turnstile dynamics were discussed to yield good explanations of the fractality of the curves depicting parameter dependencies of the conventional diffusion coefficient. This irregular parameter dependence was explained in terms of invariant densities and Takagi functions in Ref. [19]. Another large deviation analysis, which is not based on Markov partitions, was carried out by calculating higher-order cumulants, in which some exact simple recursion relations were used to explain the fractality [25].

The model used in this study is a model of diffusion applicable to quite limited conditions; it is a chaotic diffusion system created by piecewise linear mapping. Furthermore, the calculations made in this study used only the values of the parameter which allowed Markov partitioning of maps. Generally, it is not possible to analytically obtain the generalized Frobenius-Perron operator of maps in a given mapping diffusion system.

When performing calculation of the variables like the ones listed above related to deterministic diffusion (occurring from maps) as well as general diffusion, it is necessary to numerically obtain statistical structure functions from the temporal coarse graining levels of the observed variables, and to deduce from these the generalized diffusion coefficient $D_{q}$ and the generalized power spectrum $I_{q}(\omega)$. It will be difficult to plot complex parameter dependences of generalized diffusion coefficients obtained numerically. Takagi functionlike behaviors might hide behind numerical errors. In contrast, devil's staircase-like parameter dependencies of a ballistic velocity $u(\infty)$ will be relatively easily obtained for general deterministic diffusive systems. We have only to numerically find the most deviated coarse-grained velocity from the average, which is related to a single unstable periodic trajectory.

One of the open issues related to chaotic diffusion by piecewise linear mapping is to make similar calculations for maps different from those used in this study, and to examine whether or not the dependency on the parameter of the maps 
and other features revealed in this study are commonly seen in other piecewise-linear mapping systems. Another open issue is to numerically obtain statistical structure functions, generalized diffusion coefficients, etc., not only in models of mapping systems but also in actual experimental systems, with the goal of examining how the non-Gaussian characteristics will be reflected in the results.

\section{ACKNOWLEDGMENTS}

We thank Rainer Klages for many important remarks on the present paper. This study was partially supported by the 21st Century COE Program "Center of Excellence for Research and Education on Complex Functional Mechanical Systems" at Kyoto University.
[1] T. Geisel and J. Nierwetberg, Phys. Rev. Lett. 48, 7 (1984).

[2] H. Fujisaka and M. Inoue, J. Phys. Soc. Jpn. 70, 2283 (2001).

[3] R. Klages and J. R. Dorfman, Phys. Rev. Lett. 74, 387 (1995).

[4] H. Fujisaka and M. Inoue, Prog. Theor. Phys. 77, 1334 (1987).

[5] H. Fujisaka and M. Inoue, Phys. Rev. A 41, 5302 (1990).

[6] H. Fujisaka and H. Shibata, Prog. Theor. Phys. 85, 187 (1991).

[7] M. Inoue and H. Fujisaka, Phys. Rev. B 32, 277 (1985).

[8] T. Kobayashi, H. Fujisaka, and W. Just, Phys. Rev. E 47, 3196 (1993).

[9] W. Just and H. Fujisaka, Physica D 64, 98 (1993).

[10] J. R. Dorfman, An Introduction to Chaos in Nonequilibrium Statistical Mechanics (Cambridge University Press, Cambridge, England, 1999).

[11] P. Gaspard, Chaos, Scattering, and Statistical Mechanics (Cambridge University Press, Cambridge, England, 1998).

[12] P. Cvitanović et al., Chaos: Classical and Quantum (Niels Bohr Institute, Copenhagen, 2005); related URL: www.nbi.dk/ ChaosBook/

[13] S. Ito, S. Tanaka, and H. Nakada, Tokyo J. Math. 31, 279 (1983).
[14] T. Yoshida, S. Miyazaki, H. Mori, T. Kobayashi, T. Horita, and H. Hata, Prog. Theor. Phys. 82, 879 (1989).

[15] T. Yoshida and S. Miyazaki, Prog. Theor. Phys. Suppl. 99, 64 (1989).

[16] H. G. Schuster and W. Just, Deterministic Chaos, Fourth, Revised and Enlarged Edition (VCH, Weinheim, 2005).

[17] S. Grossmann and H. Fujisaka, Phys. Rev. A 26, 1779 (1982).

[18] H. Fujisaka and S. Grossmann, Z. Phys. B: Condens. Matter 48, 261 (1982).

[19] R. Klages, Deterministic Diffusion in One-Dimensional Chaotic Dynamical Systems (Wissenschaft und Technik Verlag, Berlin, 1996).

[20] R. Klages and J. R. Dorfman, Phys. Rev. E 59, 5361 (1999).

[21] R. Klages, Phys. Rev. E 65, 055203(R) (2002).

[22] E. Ott, Chaos in Dynamical Systems, 2nd ed. (Cambridge University Press, Cambridge, England, 2002).

[23] R. Klages and T. Klauß, J. Phys. A 36, 5747 (2003).

[24] Z. Koza, J. Phys. A 37, 10859 (2004).

[25] J. Groeneveld and R. Klages, J. Stat. Phys. 109, 821 (2002). 